\title{
Early Postoperative Imaging and Image-Guided Procedures on Patients with Face Transplants
}

\author{
T.C. Lee, T. Chansakul, R.Y. Huang, G.L. Wrubel, S. Mukundan, D.J. Annino, J.J. Pribaz, and B. Pomahac
}

\begin{abstract}
SUMMARY: Face transplantation is being performed with increasing frequency. Facial edema, fluid collections, and lymphadenopathy are common postoperative findings and may be due to various etiologies, some of which are particular to face transplantation. The purpose of this study was to demonstrate how postoperative imaging and image-guided minimally invasive procedures can assist in diagnosing and treating complications arising from face transplantation. Retrospective evaluation of 6 consecutive cases of face transplantation performed at Brigham and Women's Hospital between April 2009 and March 2014 was performed with assessment of postoperative imaging and image-guided procedures, including aspiration of postoperative fluid collection, lymph node biopsy, and treatment of salivary gland leak. Through these cases, we demonstrate that early postoperative imaging and image-guided procedures are key components for the management of complications following face transplantation.
\end{abstract}

$\boldsymbol{S}_{\mathrm{p}}^{\mathrm{in}}$ ince the first face transplantation in 2005, 31 cases have been performed worldwide, ${ }^{1,2}$ including the most recent case performed at our institution. Face transplantation provides functional and aesthetic restoration to severely disfigured patients with reported outcomes that surpass those achieved with conventional reconstructions. ${ }^{3-6}$ This revolutionary operation replaces damaged or missing facial units such as forehead, eyelids, nose, lips, chin, and cheeks with functional and aesthetically equivalent allografts. ${ }^{7,8}$ Face transplantation is a complex surgical procedure because patients typically have extremely complex anatomy secondary to severe injury or prior reconstructive attempts. A multidisciplinary team and extensive preoperative planning are required, for which imaging plays a vital role. As previously described in the literature, preoperative imaging aids in determining the facial defects and planning of arterial and venous anastomoses. ${ }^{9-11}$ Postoperative imaging has been useful in identifying and diagnosing postsurgical complications.

Following face transplantation, facial swelling, fluid collections, and lymphadenopathy are common. Differential diagnoses

Received July 1, 2014; accepted after revision August 23.

From the Section of Neuroradiology, Department of Radiology (T.C.L., T.C., R.Y.H., G.L.W., S.M.), Division of Otolaryngology, Department of Surgery (D.J.A.), and Division of Plastic Surgery, Department of Surgery (J.J.P., B.P.), Brigham and Women's Hospital, Boston, Massachusetts.

Please address correspondence to Thomas C. Lee, MD, Department of Radiology, Brigham and Women's Hospital, 75 Francis St, Boston, MA 02115; e-mail:

tclee@post.harvard.edu

- Indicates open access to non-subscribers at www.ajnr.org

http://dx.doi.org/10.3174/ajnr.A4141 for these findings are broad. Major differential considerations for postoperative fluid collection include seroma, hematoma, lymphocele, abscess, and salivary gland leak. Differential considerations for postoperative lymphadenopathy include rejection, inflammation, posttransplantation lymphoproliferative disease, and infection. Image-guided aspiration has readily triaged these patients with face transplants.

\section{CASE SERIES}

\section{Patient Population}

We reviewed medical records, pre- and posttransplantation imaging studies, posttransplantation image-guided procedures, and microbiology, chemistry, and pathology results for 6 consecutive face transplantations performed at the Brigham and Women's Hospital between April 2009 and March 2014. This sample comprises the first partial face transplantation, the next 4 full-face transplantations, and the sixth face transplantation, which was partial. The investigation was approved by our institutional review board and is Health Insurance Portability and Accountability Act-compliant. Written informed consent was obtained from each patient.

\section{Image-Guided Procedures}

The procedures, including fluid aspiration, lymph node biopsy, and botulinum toxin injection were performed with sonography, CT, or MR imaging guidance. The choice of imaging technique was based on which one best demonstrated the target and adjacent structures. For instance, sonography was chosen for aspiration of a superficial fluid collection, CT was chosen if a target was deep or behind a bony structure, and MR imaging was chosen in cases in which precise soft-tissue characterization was required 

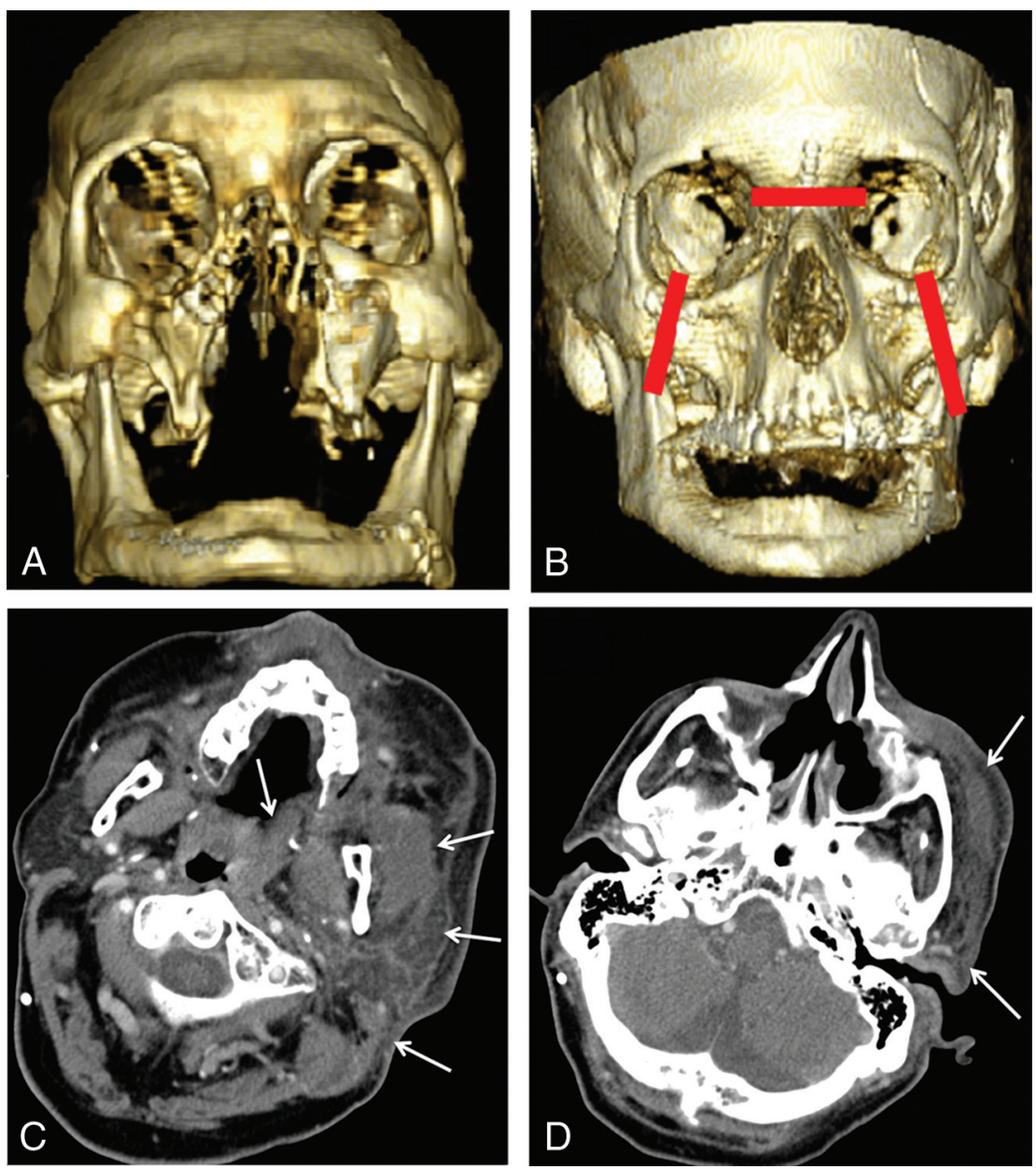

FIG 1. A, Preoperative 3D volume-rendered CT image shows posttraumatic and postreconstruction changes including absent nasal bones, anterior walls of the maxillary antra, and most of the hard palate. $B$, Postoperative 3D volume-rendered image shows fixations of the nasion and bilateral zygoma (red lines). $C$ and D, Postoperative CT shows soft-tissue swelling of the left masseter, sternocleidomastoid muscle, parotid gland, and left lateral pharyngeal wall (arrows). The degree of swelling is more prominent in recipient native tissues than in the allograft. These findings resolved with antibiotic treatment.

(eg, evaluation of a potentially displaced native salivary gland and point of salivary leak). All diagnostic study interpretations and interventional procedures shown were performed by a board-certified neuroradiologist (T.C.L.).

Image-guided aspiration and lymph node biopsy were performed with standard image-guided techniques following administration of local anesthesia. Aspiration was performed with 25-, 22-, and 20-ga needles and was sent for microbiology and chemistry testing. Lymph node biopsy was performed with an 18-ga core-needle-biopsy device and was submitted to pathology. In cases of salivary gland leak, $0.4 \mathrm{~mL}(40 \mathrm{U})$ of botulinum toxin followed by $0.1 \mathrm{~mL}$ of saline was administered in each salivary gland at the suspected point of leakage. After all needles were removed, postbiopsy imaging was performed with the same technique, to evaluate immediate postoperative complications.

\section{Patient 1}

Patient 1 underwent the first face transplantation in the Northeast or East Coast in the United States in April 2009 after sustaining massive third- and fourth-degree facial burns secondary to a highvoltage subway rail injury (Fig $1 A,-B$ ). There was significant facial swelling and erythema in the immediate postoperative period without fever. A skin-punch biopsy was performed, showing patchy perivascular chronic inflammation with foci of lymphocytic vasculopathy, consistent with early grade I acute rejection. The patient had 2 more episodes of acute rejection, both of which presented clinically with facial swelling and erythema. All bouts of rejection were responsive to pulse steroids and immunosuppressive therapy.

Five months following the transplantation, the patient presented with left orbital and nasal bridge pain and increased leftface swelling in the setting of low-grade fever preceded by a viral upper respiratory infection. CT performed at this time revealed extensive soft-tissue edema throughout the left periorbital region and left face (Fig $1 C,-D$ ), extending caudally to the level of the thyroid gland. There was no soft-tissue gas or drainable collection. The patient was placed on broad-spectrum antibiotics with rapid improvement of symptoms, favoring cellulitis or parotitis as the underlying diagnosis.

\section{Patient 2}

Patient 2 sustained a high-voltage injury to the face and left hip in November 2008 with fourth-degree facial burns and bilateral blindness (Fig 2A). Full-face transplantation was performed in 

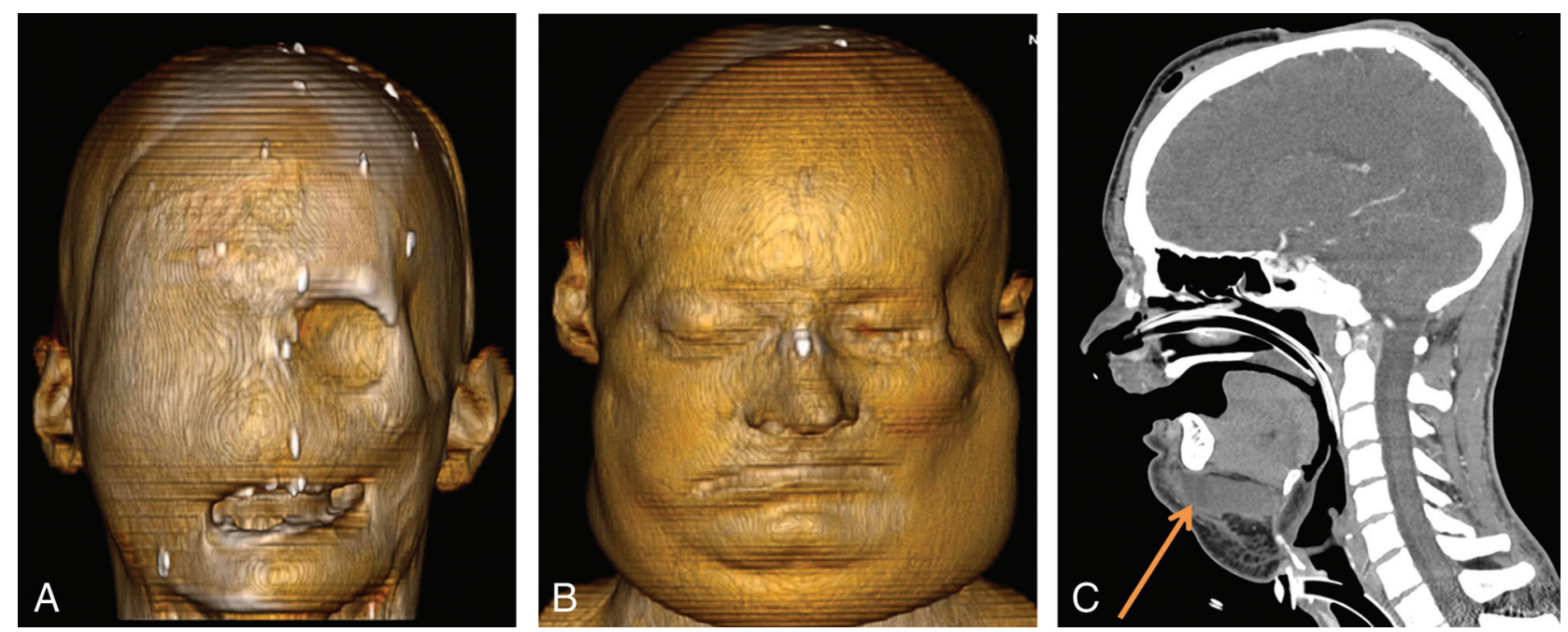

FIG 2. A, Preoperative 3D volume-rendered CT image demonstrates posttraumatic changes from a severe electrical burn. $B$, Postoperative 3D volume-rendered CT image shows facial swelling. C, Postoperative CT image demonstrates a submental fluid collection (orange arrow). Sonography-guided needle aspiration of this fluid collection yielded high amylase and low $\mathrm{pH}$, consistent with salivary collection from donor submandibular glands. The fluid collection was subsequently treated with botulinum toxin injection.
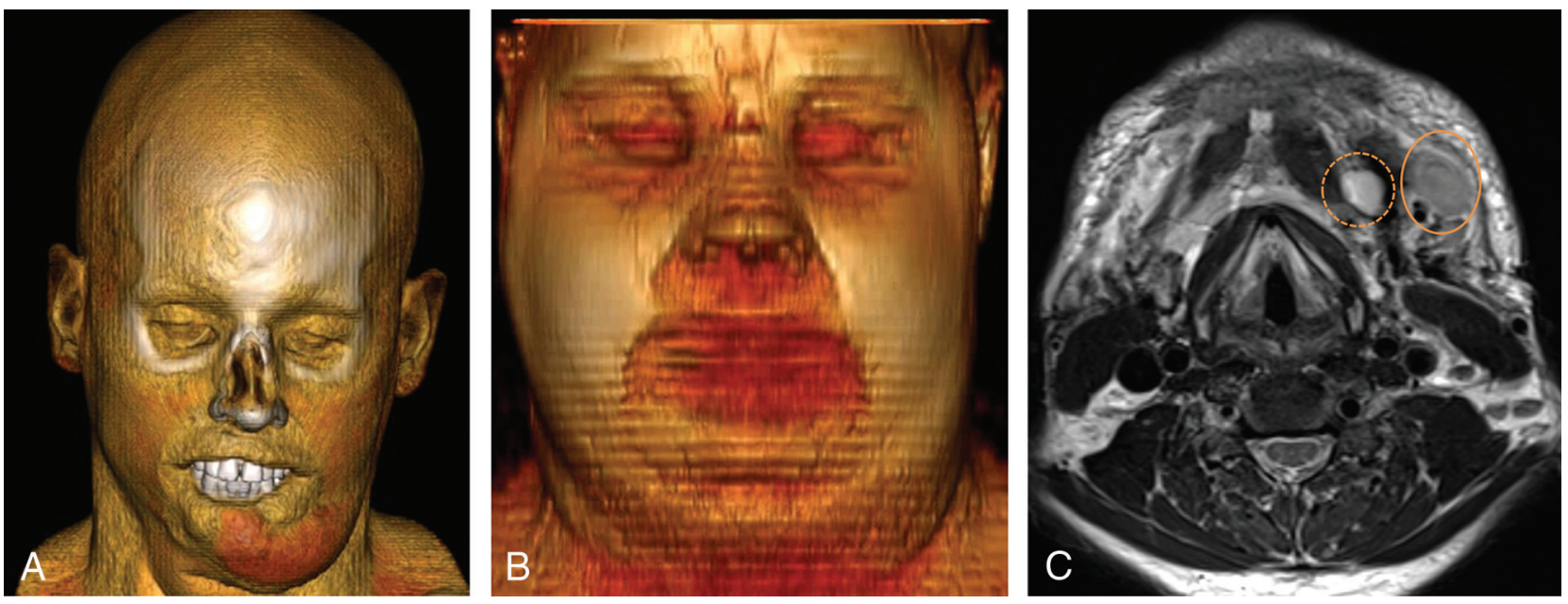

FIG 3. $A$, Preoperative 3D volume-rendered CT scan demonstrates a severely disfigured face. Note a detachable prosthetic nose. $B$, Postoperative 3D volume-rendered MR imaging performed 3 weeks following the transplantation demonstrates facial swelling. C, Postoperative T2weighted MR imaging shows a small left submandibular fluid collection, presumed seroma (circle with dotted line), and an enlarged left submandibular lymph node (circle with solid line). A skin-punch biopsy was consistent with grade I/II rejection. The patient's symptoms resolved with pulse steroid therapy.

March 2011. The immediate postoperative period was uneventful. On postoperative day 8 , the patient developed leukocytosis, facial swelling, and focal fullness along the left lower margin of the facial allograft (Fig 2B). CT performed at this time demonstrated a large submental fluid collection, a preparotid fluid collection, and multiple enlarged submandibular and submental lymph nodes. A stitch along the left lower margin of the allograft was removed, and the fluid obtained was sent for culture, which later grew Candida albicans and Pseudomonas aeruginosa. The patient was subsequently treated with cefepime and micafungin. A repeat CT performed 2 weeks following the operation showed that the submental collection was more conspicuous (Fig 2C), prompting sonography-guided aspiration, which yielded $25 \mathrm{~mL}$ of sanguineous fluid with a high amylase level and low $\mathrm{pH}$, compatible with salivary gland leak. Culture grew $C$ albicans, and the antibiotic was switched to fluconazole. Finally, both submandibular glands were injected with botulinum toxin under CT guidance (not shown), which successfully treated the salivary gland leak.

\section{Patient 3}

Patient 3 sustained high-voltage facial injury in a motor vehicle collision from a fallen telephone pole (Fig $3 A$ ). The patient did well in the immediate postoperative period but presented 3 weeks following the transplant with low-grade fever, facial swelling, and facial erythema (Fig 3B). MR imaging was performed and revealed a small left-sided submandibular fluid collection with 2 adjacent enlarged lymph nodes (Fig 3C). On physical examination, facial edema and erythema were not localized to this left submandibular fluid collection, and there was no focal tenderness at this site. Aspiration of this fluid collection was, therefore, not performed, because this was thought to represent postoperative seroma rather than infection. As with all patients post-face transplantation pre- 

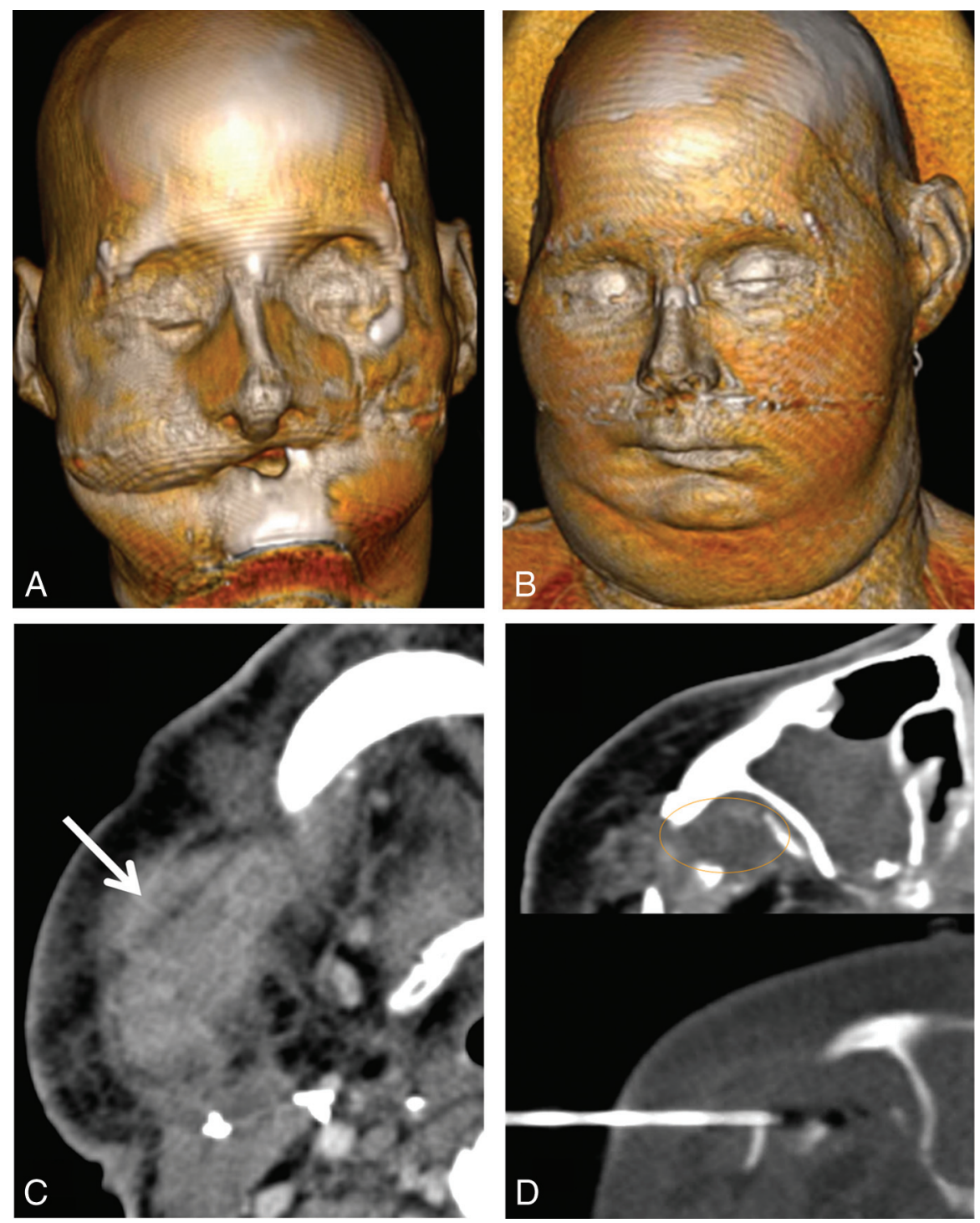

FIG 4. A, Preoperative 3D volume-rendered $C T$ image of the patient whose face was mauled by a chimpanzee. $B$, Postoperative $3 D$ volumerendered CT image obtained for persistent fever and facial swelling 4 weeks following the surgery demonstrates facial swelling. C, Postoperative CT scan shows a necrotic-appearing right submandibular lymph node (arrow). CT-guided biopsy of this lymph node showed inflammation without evidence of infection or rejection. $D$, Postoperative CT also demonstrates a small fluid collection adjacent to the native right zygoma (circle), which was aspirated under CT guidance. Culture grew Enterococcus species.

senting with facial edema, a skin-punch biopsy was performed, which, in this case, demonstrated grade I/II rejection. The patient's symptoms subsequently resolved with pulse steroid therapy.

\section{Patient 4}

Patient 4 was attacked by a chimpanzee, sustaining severe facial injuries (Fig 4A). Full-face transplantation was performed in May 2011. Four weeks following the transplantation, the patient presented with facial swelling and erythema (Fig 4B). CT revealed a 4-cm conglomerate nodal mass with low-attenuation necrosis in the right submandibular region (Fig 4C). There was also a $1.8-\mathrm{cm}$ fluid collection deep to the right zygoma with surrounding stranding and a $1.5-\mathrm{cm}$ rim-enhancing fluid collection within the left submandibular region. CT-guided biopsy of the necrotic right submandibular mass was obtained, yielding inflammation without evidence of pus, rejection, or infec- tion. CT-guided aspiration of the fluid collection deep to the native right zygoma was also performed (Fig 4D). Cultures grew Enterococcus species. The patient was placed on amoxicillin/clavulanic acid.

\section{Patient 5}

Patient 5 was a female with extensive burn injuries in 2007 after an assault with industrial lye (Fig $5 A$ ). Full-face transplantation was performed in February 2013. Two weeks following the surgery, the patient presented with facial swelling (Fig 5B). CT showed soft-tissue edema, lymphadenopathy, but no drainable fluid collection (Fig $5 C,-D$ ). Skin biopsy was consistent with grade I/II rejection, which was treated with plasma exchange.

\section{Patient 6}

Patient 6 had a remote gunshot injury to the face (Fig 6A). Partial face transplantation was performed in March 2014. In the first 

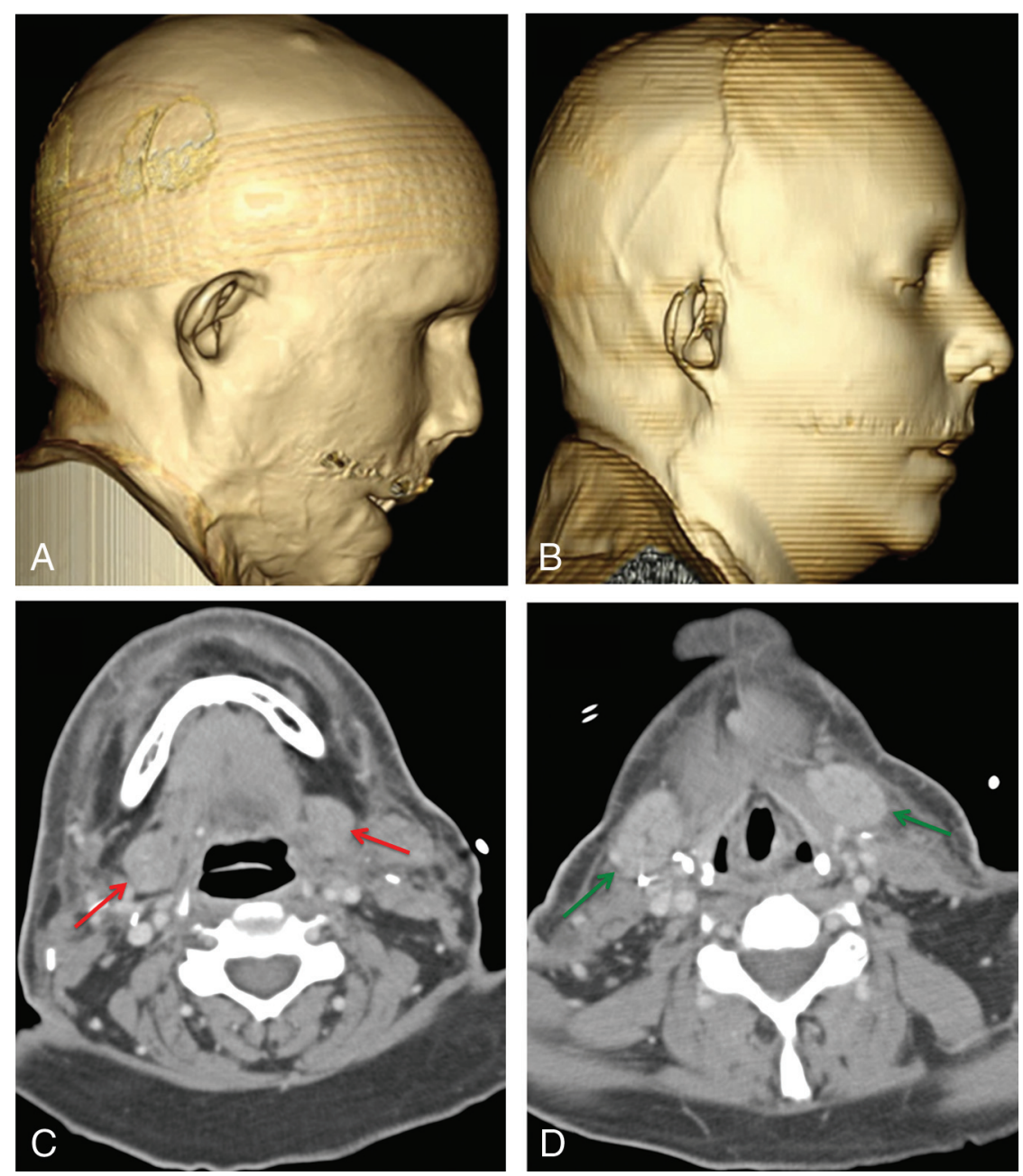

FIG 5. $A$, Preoperative 3D volume-rendered CT image demonstrates facial deformity due to a severe lye burn. $B$, Postoperative $3 D$ volumerendered $C T$ image shows mild facial swelling. $C$ and $D$, Postoperative axial $C T$ images show edema without a fluid collection. Note the normal-sized recipient submandibular glands (red arrows) and, more caudally, the symmetrically swollen bilateral donor submandibular glands (green arrows), which had been injected with botulinum toxin at the time of transplantation.

week following face transplantation, the patient developed increasing swelling of the right neck (Fig 6B). An enhanced CT scan of the head and neck revealed no rim-enhancing collection to suggest abscess; however, there was a large ill-defined fluid collection around the possibly posteriorly displaced native right submandibular gland extending to the right parotid gland (Fig 6C). Because the suspected right submandibular gland was altered in both shape and position, a differential consideration at the time included an enlarged lymph node. Review of the preoperative imaging, however, confirmed that the right submandibular gland was still present, while the left was absent, presumably due to the injury. There was no significant fluid around the 2 donor submandibular glands, which were treated with botulinum toxin injection at the time of transplantation. MR imaging-guided aspiration yielded fluid with a high amylase level, in keeping with a salivary leak (Fig 6D). MR imaging-guided botulinum toxin injection was performed at the right parotid gland and the suspected posteriorly displaced native right submandibular gland (Fig 6E, $-F$ ). Right-neck swelling subsequently resolved.

\section{DISCUSSION}

Prior literature on face transplant imaging has focused on preoperative imaging for planning of vascular anastomoses. In this article, we describe how early post-face transplantation imaging plays a vital role in identifying potential postoperative complications, particularly facial edema, fluid collections, and lymphadenopathy. Early postoperative imaging demonstrated facial edema and lymphadenopathy in all 6 cases and fluid collection in 4 cases.

Postoperative facial edema can be explained by a variety of etiologies, including lack of postsurgical lymphatic drainage, inflammation, infection, salivary gland leak, and rejection. Correlation with clinical examination and skin biopsy is helpful in making the diagnosis. At our institution, skin biopsy is obtained post-face transplantation on all patients who develop facial swelling, to evaluate rejection. Imaging with either sonography, CT, or MR imaging should be performed to evaluate the presence of a loculated fluid collection, which would favor an infectious process or salivary leak, in which case, the patient will likely benefit from image-guided aspiration for a definitive diagnosis.

Image-guided aspiration of a fluid collection was performed in 

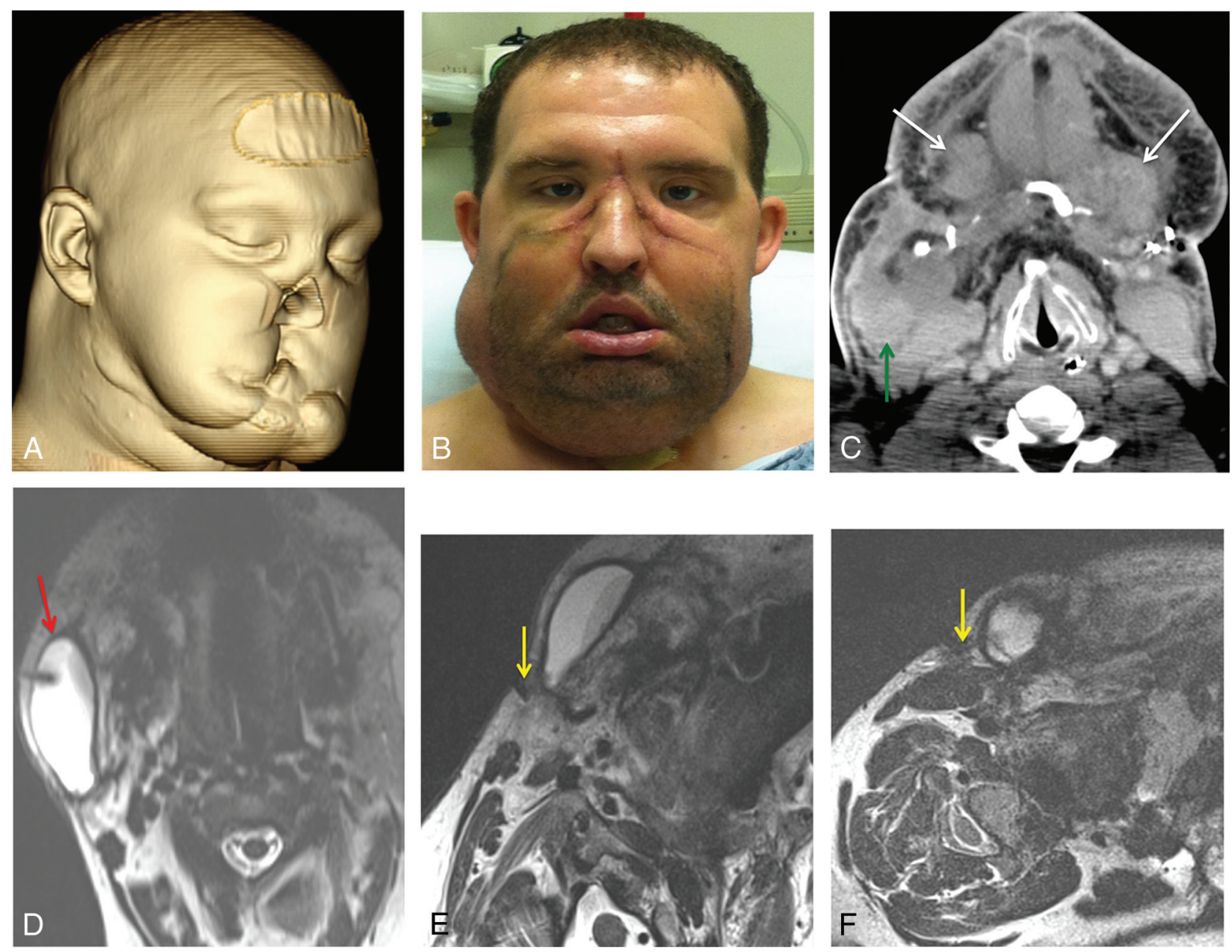

FIG 6. A, Preoperative 3D volume-rendered CT demonstrates facial deformity from a gunshot injury. B, Frontal photograph of the patient at 1 week following partial face transplantation shows right lower face and neck swelling. C, Posttransplantation CT demonstrates bilateral donor submandibular glands (white arrows), which had been injected with botulinum toxin at the time of transplantation. CT also shows a possible posteriorly displaced native right submandibular gland (green arrow), surrounded by fluid. Because the gland is altered in both shape and position, a differential consideration at this point includes an enlarged lymph node. $D$, Axial T2-weighted image obtained during MR imagingguided aspiration of the fluid collection (red arrow). Aspirated fluid had a high amylase level, in keeping with salivary leak. $E$ and $F$, Axial T2-weighted images obtained as the needles (yellow arrows) were advanced for botulinum toxin injection in the superficial right parotid gland at the site of suspected leak, determined by a tapered narrowing of the fluid collection toward the lateral aspect of the gland $(E)$, and in the suspected posteriorly displaced native right submandibular gland (F). Injection of the native right submandibular gland was targeted at its anterior portion, closest to the adjacent fluid collection.

3 patients, yielding definitive diagnoses in all cases, demonstrating either infection and/or salivary gland leak. In 2 patients in whom infection was identified, broad-spectrum antibiotics or antifungal agents could subsequently be tailored to the isolated organism.

Lymph node enlargement was observed in all patients with face transplants. Lymphadenopathy may be related to graftversus-host reaction, infection, inflammation, or lymphatic stasis. While lymphoproliferative disorders have not been observed in our patient population, Epstein Barr virus-related lymphoma has previously been reported in the literature. ${ }^{12}$ Both lymph node enlargement and facial swelling were noted to resolve in all patients 6 weeks to 2 months following surgery. In the absence of rejection and infection, this resolution in lymphadenopathy is likely secondary to restoration of postsurgical lymphatic channels.

Salivary gland leak is a surgical complication particularly re- lated to face transplantation. Patient 2, the first patient with a full transplant, was found to have a salivary gland leak, which was treated successfully with bilateral submandibular gland botulinum toxin injection. This injection has previously been used in the setting of sialorrhea secondary to Parkinson disease, stroke, head and neck cancer, and neurodegenerative disease. ${ }^{13}$ Given our success in this treatment with post-face transplantation salivary leak, all subsequent full-face transplantations performed at our institution had intraoperative submandibular gland botulinum toxin injection without image guidance as a preventive therapy. None of the subsequent cases of face transplantation experienced salivary leak except patient 6 , in whom the salivary leak may have been related to particularly difficult intraparotid facial nerve dissection and/or suspected remnants of a native right submandibular gland posteriorly displaced by the allograft. Botulinum toxin injection is directed at the site of leaking if this can be identified at imaging by the relationship of the fluid collection to the 
gland. For instance, in patient 6 , there appeared to be a tapered narrowing of the fluid collection toward the lateral aspect of the right superficial parotid gland, and botulinum toxin injection was targeted at this site.

\section{CONCLUSIONS}

Face transplantation is a complex procedure, which is being performed with increasing frequency. Early postoperative face transplantation complications are varied, including rejection, infection, abscess, lymphocele, hematoma, and salivary gland leak. Postoperative sonography, CT, or MR imaging can easily identify discrete fluid collections in the setting of postoperative facial swelling. Once a fluid collection is identified, image-guided aspiration is a minimally invasive technique, which provides a rapid definitive diagnosis that guides therapeutic management. Image-guided botulinum toxin injection has been effective as a preventive and therapeutic procedure for salivary leak.

Disclosures: T.C. Lee_UNRELATED: Payment for Development of Educational Presentations: Elsevier, Comments: Atlas coauthor. Srinivasan Mukundun-RELATED: Grant: Department of Defense, ${ }^{*}$ Comments: sponsors portions of face transplant program; UNRELATED: Grants/Grants Pending: National Institutes of Health, * Comments: received shared instrument grant to buy a preclinical 7T MRI scanner; Payment for Development of Educational Presentations: Elsevier, Comments: Atlas textbook coauthor; Stock/Stock Options: consultant, Comments: Alzeca, MKT, and Marval. I received stock options for consultant work. These are separate startup companies without a clinical product. D.J. Annino-RELATED: Grant: Department of Defense, * Comments: partial salary support from research contract, \#W911QY-09C-0216. J.J. Pribaz-RELATED: Grant: Department of Defense, ${ }^{*}$ Comments: partial salary support from research contract, \#W911QY-09-C-0216. B. Pomahac-RELATED: Grant: Department of Defense, ${ }^{*}$ Comments: partial salary support from research contract, \#W911QY-09-C-0216. *Money paid to the institution.

\section{REFERENCES}

1. Altman LK. An about-face on a risky transplant. New York Times. April 29, 2014:D1

2. Khalifian S, Brazio PS, Mohan R, et al. Facial transplantation: the first 9 years. Lancet 2014;384:2153-63

3. Lantieri L, Meningaud JP, Grimbert P, et al. Repair of the lower and middle parts of the face by composite tissue allotransplantation in a patient with massive plexiform neurofibroma: a 1-year follow-up study. Lancet 2008;372:639-45

4. Petruzzo P, Testelin S, Kanitakis J, et al. First human face transplantation: 5 years outcomes. Transplantation 2012;93:236-40

5. Pomahac B, Pribaz J, Eriksson E, et al. Restoration of facial form and function after severe disfigurement from burn injury by a composite facial allograft. Am J Transplant 2011;11:386-93

6. Pomahac B, Pribaz J, Eriksson E, et al. Three patients with full facial transplantation. N Engl J Med 2012;366:715-22

7. Barret JP, Gavalda J, Bueno J, et al. Full face transplant: the first case report. Ann Surg 2011;254:252-56

8. Jeung IS, Lee S, Kim HS, et al. Effect of botulinum toxin a injection into the salivary glands for sialorrhea in children with neurologic disorders. Ann Rehabil Med 2012;36:340-46

9. Sisk GC, Kumamaru KK, Schultz K, et al. Cine computed tomography angiography evaluation of blood flow for full face transplant surgical planning. Eplasty 2012;12:e57

10. Soga S, Pomahac B, Wake N, et al. CT angiography for surgical planning in face transplantation candidates. AJNR Am J Neuroradiol 2013;34:1873-81

11. Soga S, Wake N, Bueno EM, et al. Noninvasive vascular images for face transplant surgical planning. Eplasty 2011;11:e51

12. Siemionow M, Ozturk C. Face transplantation: outcomes, concerns, controversies, and future directions. J Craniofac Surg 2012;23:254-59

13. Lengelé BG. Current concepts and future challenges in facial transplantation. Clin Plas Surg 2009;36:507-21 